\title{
53. Staining of the Nemaline Rod by Fluorescent Antibody against 10S-actinin
}

\author{
By Hideo Sugita, *) Tomoh MaSaKI, **) Setsuro EbashI, ${ }^{* *)}$ \\ and Carl M. PeArson ***) \\ (Comm. by Shigeo OKinaka, M. J. A., March 12, 1974)
}

Nemaline myopathy was first described by Shy et al. ${ }^{1)}$ in 1963 as a congenital nonprogressive disease of skeletal muscle which was associated with the presence of rod-like structures, predominantly in type I fibers. Nemaline-like rods, however, have been found in various neuromuscular disorders other than congenital nemaline myopathy, suggesting that rod formation may be a rather common reaction of muscle cells under some pathological conditions.

There has been a lot of controversy concerning the chemical composition of nemaline rods. Tropomyosin, ${ }^{2)}$ actin or both ${ }^{3)}$ were listed as the candidates, but a clear conclusion has not been drawn yet.

On the basis of chemical and immunological studies we have reported ${ }^{4)}$ that neither actin, $\alpha$-actinin, nor tropomyosin are contained within nemaline rods, contrary to some previous speculations about their make-up. In the present communication, we will present the evidence that the $10 \mathrm{~S}$ component in crude $\alpha$-actinin preparation (10S-actinin) is a constituent of the nemaline rod as well as the Z-band.

Materials and methods. The materials used for the experiment were the same as described in our previous paper. ${ }^{4)}$

The 10S-actinin was extracted from human cadavers free from neuromuscular disorders by the method of Masaki and Takaiti, ${ }^{5)}$ and injected into rabbits individually and repeatedly in conjuction with Freund's complete adjuvant.

The antibody thus obtained was labeled with fluorescein isothiocyanate (FITC) for fluorescence study.

Results. The glycerinated nemaline rod-containing muscle fibers and myofibrils were stained with FITC-labeled antibody against human 10S-actinin. When the sarcolemmal membrane was intact, it was often difficult for the antibody to penetrate into the inside of the

*) Department of Neurology, Institute of Brain Research. Faculty of Medicine, University of Tokyo.

**) Department of Pharmacology, Faculty of Medicine, University of Tokyo. fornia.

***) Department of Medicine, UCLA School of Medicine, Los Angeles, Cali- 

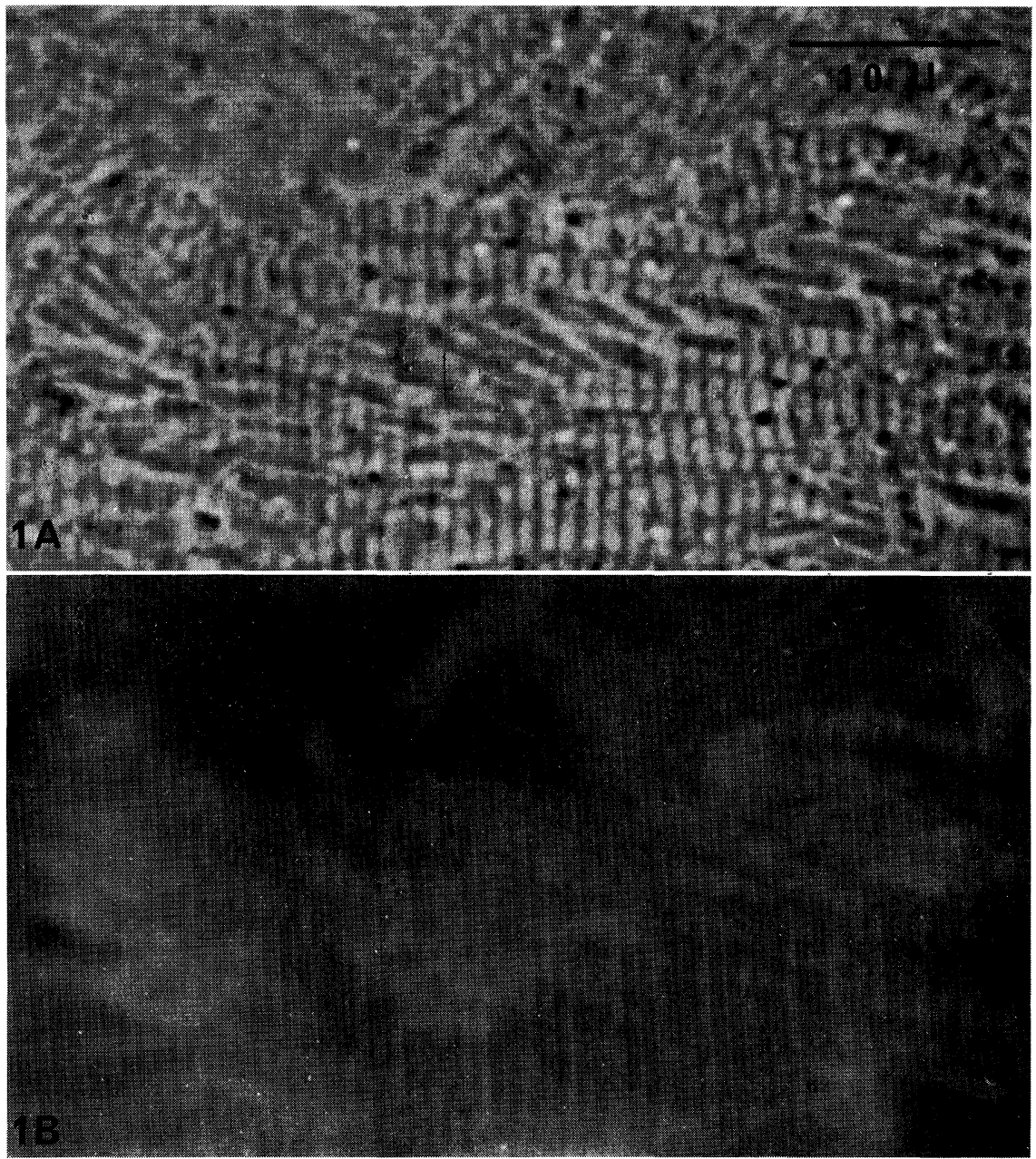

Fig. 1. Micrographs of a nemaline muscle fiber stained with the fluorescein isothiocyanate-labeled anti-10S-actinin antibody.

A. Phase microscope: Dense homogeneous bar-like structures of $2-4 \mu \mathrm{m}$ in their lengths, lying perpendicular or oblique to the striations, are the nemaline rods. Since the muscle fiber has been treated with HasselbachSchneider's solution to remove myosin and then with the antibody, its original striation patterns are not observable. The striations in this photograph cannot be identified with either the Z-band or M-line, unless they are referred to those in the fluorescence micrograph below.

B. Fluorescence microscope: All the rods under the phase microscope are stained with the anti-human-10S-actinin antibody. Among the two striations, the thicker one is the Z-band and the thinner one M-line; the latter is stained with the contaminated anti-human-M-protein antibody. 
muscle fibers. In these cases the following steps were followed: (a) staining by fluorescent protein, (b) incubation with HasselbachSchneider's solution, (c) restaining by the fluorescent antibody.

It was demonstrated that the FITC-labeled antibody against $10 \mathrm{~S}-$ actinin distinctly stained the nemaline rods in addition to staining the Z-band regions and middle of the A-band as illustrated in Figure (as for the staining of the middle of the A-band, see the Discussions). Hence, the composition of the nemaline rod as being comprised partially, or totally, of 10S-actinin was finally disclosed.

Discussions. As reported in the previous communication, ${ }^{4)}$ we failed to demonstrate the binding to rods of antibody against $\alpha$ actinin or tropomyosin, or the attachment of H-meromyosin to the rods. On the other hand the presence of 10S-actinin in the rods has been suggested because of their sensitivity to trypsin. ${ }^{4)}$ In this article we succeeded in demonstrating the binding to the rods and Z-band regions of the FITC-labeled antibody against human 10S-actinin. As stated in another paper, ${ }^{6}$ ) the antibody we prepared also contained antibodies against the M-protein and $\alpha$-actinin besides 10S-actinin. The staining at the middle of the A-band is considered to be due to the antibody against M-protein which contaminated the antiserum against 10S-actinin, as is the case in $\alpha$-actinin. However, the possibility that the nemaline rod would contain $\alpha$-actinin and/or M-protein is ruled out by failure in staining the rods with antibodies against M-protein as well as $\alpha$-actinin.

Therefore, we can conclude that the rods contain 10S-actinin. No evidence has been presented to indicate the presence of other structural proteins in the rods.

As shown in previous papers, ${ }^{6)}$ no distinction in amino acid composition, peptide map, and electrophoretic mobility in the presence of SDS and $\beta$-mercaptoethanol has been observed between actin and 10 S-actinin. In spite of our success in producing antibody against 10S-actinin, however, all attempts have so far failed to obtain antibody against actin. Any types of actin, including fibrous, globular, alkaline-denatured and trypsin-digested fragments do not react with anti-10S-actinin antibody, whereas alkaline-denatured as well as trypsin-digested forms of 10 S-actinin easily react with the antibody. Furthermore, 10S-actinin has a definitely higher rate of turnover than actin.7) These facts exclude the possibility that 10S-actinin is a type of denatured actin.

Further immunochemical investigation suggests that 10S-actinin is a constituent not only of the Z-band but also of the entire thin filament. ${ }^{6}$ ) It is conceivable that 10 S-actinin is a kind of isomer of actin and forms the main strands of the thin filament together with 
actin.

Conclusion. On the basis of the immunological studies, it is concluded that the 10S-component in crude $\alpha$-actinin preparation (10S-actinin) is a constituent of the nemaline rod as well as the Z-band region.

Acknowledgement. We wish to express our cordial thanks to Prof. Y. Toyokura for his encouragement and to Dr. T. Shimizu for his cooperation. Our thanks are also due to Miss N. Shippo and T. Hirai for their technical assistance. This work was supported by grants from the Muscular Dystrophy Associations of America, Inc., the Ministry of Health and Welfare (No.216) and by a USPHS grant GM 15795.

\section{References}

1) Shy, G. M., Engel, W. K., Somers, J. E., and Wanko, T.: Brain, 86, 793 (1963).

2) Price, H. M., Gordon, G. B., Pearson, C. M., Munsat, T. L., and Blumberg, M. J.: Proc. Nat. Acad. Sci., 54, 1938 (1965).

3) Engel, A. G., and Gomez, M. R.: J. Neuropath, Exper. Neurol., 26, 601 (1967).

4) Sugita, H., Masaki, T., Ebashi, S., and Pearson, C. M.: p. 298, Basic Research in Myology, Proc. IInd Internat. Congr. on Muscle Diseases, Perth, 22-26, Nov. 1971.

5) Masaki, T., and Takaiti, O.: J. Biochem., 66, 637 (1969).

6) Sugita, H., Masaki, T., and Ebashi, S.: J. Biochem., 75, 671 (1974).

7) Koizumi, T.: J. Biochem., in press. 